\title{
Carotid Artery Angioplasty and Stenting for Carotid Stenosis: A Single-Center Experience from Saudi Arabia
}

\author{
Hussein Algahtani, MD, FRCPC, MMed ${ }^{1}$, Bader Shirah, MD², Abdullah S. Alghamdi, MD², \\ Ahmad Subahi, $\mathrm{PhD}^{3}$, Saeed A. Alqahtani, $\mathrm{MD}^{4}$, Abdulrahman Alshamy, $\mathrm{MD}^{1}$ \\ ${ }^{1}$ King Abdulaziz Medical City, King Saud bin Abdulaziz University for Health Sciences, Jeddah, Saudi Arabia \\ ${ }^{2}$ King Abdullah International Medical Research Center, King Saud bin Abdulaziz University for Health Sciences, Jeddah, Saudi Arabia \\ ${ }^{3}$ King Saud bin Abdulaziz University for Health Sciences, Jeddah, Saudi Arabia \\ ${ }^{4}$ College of Medicine, King Khalid University, Abha, Saudi Arabia
}

Purpose: Atherosclerotic stenosis of the extracranial carotid artery accounts for approximately $20 \%$ of all strokes. Both carotid artery endarterectomy and carotid artery angioplasty with stenting (CAAS) are recommended for symptomatic patients with $50 \%$ or more stenosis or asymptomatic patients with $70 \%$ or more stenosis. CAAS is under-reported in Saudi Arabia, as evidenced by a thorough literature search. In this article, we aim to share our experience of CAAS to call for the necessity of conducting more research on stroke and emphasize the local need of utilizing more endovascular treatments like CAAS.

Materials and Methods: A retrospective single-center observational study was conducted at King Abdulaziz Medical City in Jeddah, Saudi Arabia. The inclusion criteria consisted of all adult patients (18 years and above) with carotid stenosis who were treated with CAAS.

Results: A total of 16 patients were included in the study. The mean age of the participants was 66.9 \pm 13.5 years (range 30-87 years). All patients were symptomatic (had a previous stroke or transient ischemic attack). The procedure was successful in 14 patients (87.5\%), while it failed in 2 patients (12.5\%) due to technical reasons. All patients had no stroke or myocardial infarction within 30 days of the procedure.

Conclusion: Despite the advancement in medicine with free healthcare services in Saudi Arabia, the interventional procedures for secondary prevention of strokes are underutilized. Collaboration between different hospitals will be extremely helpful since few centers in each city are providing such treatments by an expert neurointerventionist and/or strokologist. The good selection of candidates, optimal management of comorbid conditions, and multidisciplinary care may improve outcomes and reduce mortality.

Key Words: Angioplasty; Stent; Carotid stenosis; Stroke; Saudi Arabia

\section{Correspondence to: Hussein Algahtani, MD, FRCPC, MMed \\ King Abdulaziz Medical City/ King Saud bin Abdulaziz University for Health Sciences, P.O. Box: 12723, Jed- dah 21483, Saudi Arabia Tel: +966556633130 \\ E-mail: halgahtani@hotmail.com}

Received: February 24, 2020

Revised: August 15, 2020 Accepted: August 17, 2020
Copyright $\odot 2020$ Korean Society of Interventional Neuroradiology This is an Open Access article distributed under the terms of the Creative Commons Attribution Non-Commercial License (http://creativecommons.org/licenses/by-nc/4.0) which permits unrestricted non-commercial use, distribution, and reproduction in any medium, provided the original work is properly cited. 


\section{INTRODUCTION}

Strokes are the second leading cause of death and a major cause of disability worldwide.' In Saudi Arabia, around 6,000 strokes occur annually; of them, 1,500 end in death and 3,500 leave the patient with significant disability. ${ }^{2}$ Worldwide, atherosclerotic stenosis of the extracranial carotid artery accounts for approximately $20 \%$ of all strokes with no statistics or data coming from Saudi Arabia. ${ }^{3}$ The diagnosis and treatment of carotid artery disease have improved significantly over the past 2 decades with the emergence of medical treatment and carotid revascularization procedures. ${ }^{4}$ Both carotid artery endarterectomy (CEA) and carotid artery angioplasty with stenting (CAAS) are recommended for symptomatic patients with $50 \%$ or more stenosis or asymptomatic patients with $70 \%$ or more stenosis. ${ }^{5}$ CEA has some limitations due to surgical complications, patients' comorbid conditions making surgery risky, and unfavorable neck anatomy. ${ }^{6}$ Therefore, CAAS has been developed to avoid these complications and provide a minimally invasive and safer procedure. ${ }^{7}$ Although there are concerns associated with CAAS because of periprocedural complications such as strokes, technological improvement with advances in catheters has made this concern less important. ${ }^{8}$ CAAS is under-reported in Saudi Arabia, as evidenced by a thorough literature search. There are very few studies on carotid artery disease, CEA, and CAAS. These studies have several limitations including small sample sizes, retrospective studies with data extracted from hospital files, and a lack of standardization in the documentation. In addition, these studies originated from a single tertiary care center, which makes them not generalizable to the Saudi general population., ${ }^{9,10}$ In this article, we aim to share our experience of CAAS to call for the necessity of conducting more research on stroke and emphasize a local need for utilizing more endovascular treatments like CAAS.

\section{MATERIALS AND METHODS}

A retrospective single-center observational study was conducted at King Abdulaziz Medical City in Jeddah, Saudi Arabia. The collected data covered the procedures, which were done over a period of 5 years from January 2012 to December 2017. The inclusion criteria consisted of all adult (18 years and above) patients with carotid stenosis who were treated with CAAS. Patients whose data were incomplete were ex- cluded.

Carotid stenosis was defined as $\geq 70 \%$ stenosis in the internal carotid artery on ultrasound and/or computed tomography angiography of the carotid artery. The procedure for carotid angioplasty in our hospital was done under conscious sedation with an aseptic technique. All patients received dual antiplatelet using aspirin $325 \mathrm{mg}$ and clopidogrel $75 \mathrm{mg}$ daily. The dual antiplatelet therapy was started at least 7 days before the procedure to ensure maximum platelet inhibition. All carotid stent placements were performed in a dedicated angiography suite (Artis Zee; Siemens, Erlangen, Germany). Local anesthesia using lidocaine 1 percent was done on the right femoral artery area, followed by the insertion of a short $10 \mathrm{~cm}$ Terumo (Terumo Medical, Tokyo, Japan) 8-French sheath in the common femoral artery. After insertion, a common carotid artery angiogram was done to determine the location of the stenosis and the technical feasibility of stenting.

After administration of 5,000 international units of heparin, we performed an exchange for a stiff guidewire, and then an 8-French guiding-catheter (Neuron Max 0.088; Penumbra Inc., Alameda, CA, USA) was placed in the common carotid artery. Carotid artery stenting was performed with the use of a carotid Wallstent and a FilterWire EZ (Boston Scientific Corporation, Natick, MA, USA). Pre-stenting dilatation was performed with $1 \mathrm{mg}$ atropine. We used pre-stenting percutaneous transluminal angioplasty balloon dilatation using an Aviator Plus 4-7 mm diameter balloon (Cordis Corp., Miami, $\mathrm{FL}, \mathrm{USA}$ ). Occasionally, we performed angioplasty after carotid stenting if the residual stenosis was more than 50 percent. After the procedure, all patients continued on dual antiplatelets for 6 months. They then switched to single antiplatelet using aspirin $81 \mathrm{mg}$ daily.

Data were obtained from the patients' files and collected on the data collection sheets. Simple descriptive statistical tests, including mean and standard deviation, frequency, and percentage, were utilized. Data analysis was done using the Statistical Package for Social Sciences (SPSS) version 23.0 (IBM Co., Armonk, NY, USA). The significance level was predetermined at a P level $<0.05$ for all tests. This study was approved by the Institutional Review Board (IRB) of King Abdullah International Medical Research Center under IRB number IRBC/0676/18. 


\section{RESULTS}

A total of 16 patients were included in the study. Twelve patients were male (75.0\%), while 4 were female (25.0\%). The mean age of the participants was $67 \pm 14$ years (range 30-87 years). Family history was negative for carotid artery stenosis for all patients. All patients had multiple comorbidities such as diabetes, hypertension, and dyslipidemia. All patients were symptomatic (had a previous stroke). The radiological modality of diagnosis was ultrasound in 11 patients (68.8\%) and computed tomography angiography in 5 patients (31.3\%). The procedure was successful in 14 patients (87.5\%), while it failed in 2 patients (12.5\%) due to technical reasons. Causes of failure were complete occlusion of the artery and aortoiliac occlusion (Leriche syndrome). Fifteen patients had no complications (93.8\%), while 1 patient (6.3\%) had instent thrombosis and symptomatic showering of thrombi (intermittent weakness and facial droop with no changes on brain computed tomography scan) likely due to insufficient platelet inhibition despite being on aspirin $325 \mathrm{mg}$ and clopidogrel $75 \mathrm{mg}$ daily 7 days before the procedure. All patients had no stroke or myocardial infarction within 30 days of the procedure. The mean length of follow-up was $32 \pm 18$ months (range 6-60 months). Seven patients (43.8\%) were still alive during our review of the cases, 3 patients died (18.8\%), and 6 patients (37.5\%) were lost to follow-up. The functional outcome (such as the modified Rankin score) before and after the patient's procedure was not part of the protocol for this procedure (Tables 1, 2, and Fig. 1).

\section{DISCUSSION}

Despite advancements in medicine with free healthcare services in Saudi Arabia, interventional procedures for secondary prevention of strokes are underutilized. This was demonstrated clearly in the low number of procedures done during a 5-year period. Our center is considered a tertiary care center and one of the few centers performing this procedure. This low number could be either due to low referral, late presentation, or underutilization of this procedure. Explaining the limitations of performing this procedure in Saudi Arabia requires further investigations and research. Our speculation is that several factors are contributing including a lower incidence of atherosclerotic large vessel disease in Saudi Arabia, under-investigation of carotid artery pathology in patients presenting with stroke or TIA, late presentation in patients with complete occlusion of the artery, and lack of experienced neurointerventionists and/or strokologists. Collaboration between different hospitals will be extremely helpful since few centers in each city provide such treatments by expert neurointerventionists and/or strokologists. The good selection of candidates, optimal management of comorbid conditions, and multidisciplinary care with cardiology and cardiac services utilization may improve outcomes

Table 1. Summary of the clinical data of patients in our study

\begin{tabular}{|c|c|}
\hline Variable & Value \\
\hline Mean age (range 30-87 y) & $67 \pm 14 y$ \\
\hline Male & $12(75)$ \\
\hline Female & $4(25)$ \\
\hline Male to female ratio & $3: 1$ \\
\hline \multicolumn{2}{|l|}{ Family history of carotid artery stenosis } \\
\hline Positive & $0(0)$ \\
\hline Negative & $16(100)$ \\
\hline \multicolumn{2}{|l|}{ Comorbidities } \\
\hline Present & $16(100)$ \\
\hline Absent & $0(0)$ \\
\hline \multicolumn{2}{|l|}{ Presentation } \\
\hline Symptomatic & $16(100)$ \\
\hline Asymptomatic & $0(0)$ \\
\hline \multicolumn{2}{|l|}{ Radiological modality of diagnosis } \\
\hline Ultrasound & $11(69)$ \\
\hline Computed tomography angiography & $5(31)$ \\
\hline \multicolumn{2}{|l|}{ Outcome of the procedure } \\
\hline Success & $14(87.5)$ \\
\hline Failure & $2(12.5)$ \\
\hline \multicolumn{2}{|l|}{ Complications } \\
\hline Present & $1(6)$ \\
\hline Absent & $15(94)$ \\
\hline \multicolumn{2}{|l|}{$\begin{array}{l}\text { Stroke or myocardial infarction within } 30 \text { days } \\
\text { of the procedure }\end{array}$} \\
\hline Present & $0(0)$ \\
\hline Absent & $16(100)$ \\
\hline Mean length of follow-up (range 6-60 mo) & $31.5 \pm 18.1 \mathrm{mo}$ \\
\hline \multicolumn{2}{|l|}{ Follow-up } \\
\hline Alive & $7(44)$ \\
\hline Death & $3(19)$ \\
\hline Unknown & $6(37)$ \\
\hline
\end{tabular}

$\overline{\text { Values are presented as mean } \pm \text { standard deviation or number (\%). }}$ 
Table 2. Clinical details of the study participants

\begin{tabular}{|c|c|c|c|c|c|c|c|c|c|}
\hline Patient no. & Age (y) & Sex & Comorbidities & Presentation & $\begin{array}{l}\text { Radiological } \\
\text { modality }\end{array}$ & $\begin{array}{l}\text { Procedure } \\
\text { outcome }\end{array}$ & Complications & $\begin{array}{l}\text { Length of } \\
\text { follow-up }\end{array}$ & Outcome \\
\hline Patient 1 & 68 & Male & $\begin{array}{l}\text { Diabetes, } \\
\text { hypertension, and } \\
\text { dyslipidemia }\end{array}$ & Stroke & Ultrasound & Failure & $\begin{array}{l}\text { In-stent } \\
\text { thrombosis and } \\
\text { showering of } \\
\text { thrombi }\end{array}$ & 2 years & Death \\
\hline Patient 2 & 83 & Male & $\begin{array}{l}\text { Hypertension and } \\
\text { dyslipidemia }\end{array}$ & Stroke & Ultrasound & Success & None & 5 years & Survival \\
\hline Patient 3 & 87 & Male & $\begin{array}{l}\text { Hypertension and } \\
\text { obesity }\end{array}$ & Stroke & $\begin{array}{l}\text { Computed } \\
\text { tomography } \\
\text { angiography }\end{array}$ & Success & None & 5 years & Survival \\
\hline Patient 4 & 73 & Male & $\begin{array}{l}\text { Hypertension and } \\
\text { dyslipidemia }\end{array}$ & Stroke & Ultrasound & Success & None & 2 years & Unknown \\
\hline Patient 5 & 72 & Male & $\begin{array}{l}\text { Diabetes, } \\
\text { hypertension, and } \\
\text { dyslipidemia }\end{array}$ & Stroke & Ultrasound & Success & None & 4 years & Death \\
\hline Patient 6 & 77 & Male & $\begin{array}{l}\text { Diabetes and } \\
\text { dyslipidemia }\end{array}$ & Stroke & Ultrasound & Success & None & 1.5 years & Unknown \\
\hline Patient 7 & 65 & Male & $\begin{array}{l}\text { Diabetes, } \\
\text { hypertension, and } \\
\text { dyslipidemia }\end{array}$ & Stroke & $\begin{array}{l}\text { Computed } \\
\text { tomography } \\
\text { angiography }\end{array}$ & Success & None & 6 months & Unknown \\
\hline Patient 8 & 64 & Male & $\begin{array}{l}\text { Diabetes and } \\
\text { hypertension }\end{array}$ & Stroke & Ultrasound & Success & None & 1 year & Survival \\
\hline Patient 9 & 64 & Male & $\begin{array}{l}\text { Diabetes and } \\
\text { hypertension }\end{array}$ & Stroke & $\begin{array}{l}\text { Computed } \\
\text { tomography } \\
\text { angiography }\end{array}$ & Success & None & 2 years & Survival \\
\hline Patient 10 & 62 & Male & $\begin{array}{l}\text { Diabetes, } \\
\text { hypertension, and } \\
\text { dyslipidemia }\end{array}$ & Stroke & Ultrasound & Success & None & 2 years & Unknown \\
\hline Patient 11 & 66 & Male & $\begin{array}{l}\text { Diabetes, } \\
\text { hypertension, and } \\
\text { dyslipidemia }\end{array}$ & Stroke & $\begin{array}{l}\text { Computed } \\
\text { tomography } \\
\text { angiography }\end{array}$ & Failure & None & 2 years & Unknown \\
\hline Patient 12 & 48 & Male & $\begin{array}{l}\text { Diabetes, } \\
\text { hypertension, and } \\
\text { dyslipidemia }\end{array}$ & Stroke & Ultrasound & Failure & None & 4 years & Survival \\
\hline Patient 13 & 67 & Female & $\begin{array}{l}\text { Diabetes, } \\
\text { hypertension, and } \\
\text { dyslipidemia }\end{array}$ & Stroke & Ultrasound & Success & None & 3 years & Survival \\
\hline Patient 14 & 79 & Female & $\begin{array}{l}\text { Hypertension and } \\
\text { dyslipidemia }\end{array}$ & Stroke & Ultrasound & Success & None & 5 years & Survival \\
\hline Patient 15 & 66 & Female & $\begin{array}{l}\text { Diabetes and } \\
\text { dyslipidemia }\end{array}$ & Stroke & Ultrasound & Success & None & 1 year & Unknown \\
\hline Patient 16 & 30 & Female & $\begin{array}{l}\text { Nasopharyngeal } \\
\text { carcinoma and } \\
\text { radiation therapy }\end{array}$ & Stroke & $\begin{array}{l}\text { Computed } \\
\text { tomography } \\
\text { angiography }\end{array}$ & Success & None & 2 years & Death \\
\hline
\end{tabular}

and reduce mortality.

In our study, the mean age of our patients was $67 \pm 14$ years, with 2 patients above the age of 80 years. Those octogenarians performed well even after 5 years of follow-up. One of our patients had the procedure at the age of 30 who unfortunately died from an underlying nasopharyngeal carcinoma, unrelated to her carotid artery disease or her previous symptomatic stroke. It is well known that radiation therapy 


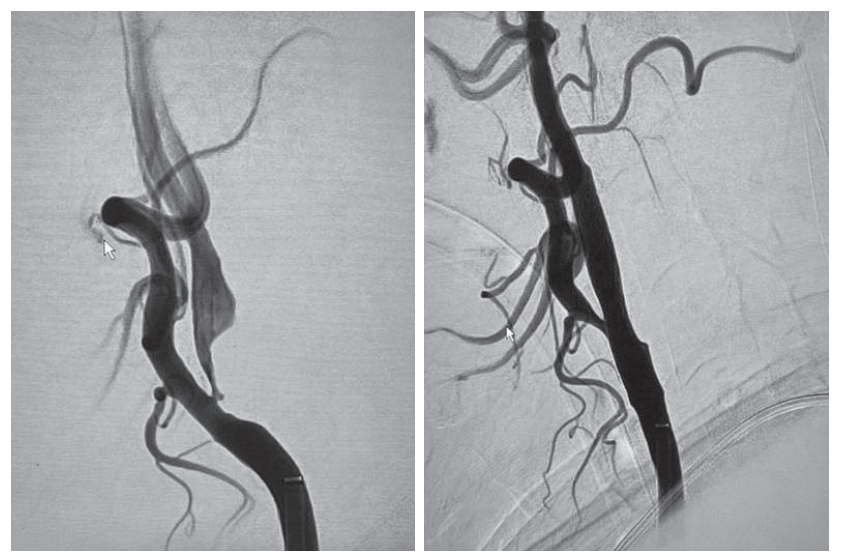

Fig. 1. A lateral view of a cerebral angiogram of the right common carotid artery post angioplasty and carotid stent placement of an 83-year-old male with diabetes and hypertension (patient 2 in Table 2).

is harmful to the endothelium of the vascular structure. This patient had radiation-induced carotid stenosis, which was symptomatic. Thus, she was treated with carotid stenting. Another patient had the procedure at the age of 48 , which failed due to the presence of aortoiliac occlusion (Leriche syndrome). The impact of age has been a subject of debate for many years, particularly those above the age of 80 years. Age per se is not an exclusion for CAAS. Elderly patients should be evaluated for the complex arch and carotid anatomy and heavy calcification of the target lesion. In addition, elderly patients above the age of 80 years with asymptomatic severe carotid stenosis and life expectancy less than 3 years should not undergo prophylactic CAAS since these patients may not live long enough to derive benefit. In younger patients with severe carotid artery disease requiring CAAS, thorough investigations should be performed to exclude etiologies like Moyamoya Disease and diseases causing technical difficulties when performing the procedure. ${ }^{8}$

In our study, 4 patients (25.0\%) were female with excellent results and no periprocedural complications. The low number of female patients who had the procedure can be explained by the results of a pooled analysis from the North American Symptomatic Carotid Artery Endarterectomy Trial ${ }^{11}$ and the European Carotid Surgery Trial, ${ }_{1}^{12}$ which showed that medical therapy is superior to surgical intervention except for those patients with additional risk factors (age $>70$ years, severe hypertension, history of myocardial infarction, or hemispheric events). Women have an increased 30-day risk of death and higher perioperative complications..$^{13}$

In our study, there was no documented family history of carotid artery disease or strokes related to the carotid system. Although the sample size is small, the results of our study indicate no predisposing genetic factors involved, and the cause of the disease is most likely related to risk factors. Unfortunately, we did not ask about the family history of strokes of any cause or coronary artery disease. In a study performed by Khaleghi et al. ${ }^{14}$, a family history of stroke or congestive heart disease was independently associated with carotid artery disease. In addition, siblings' history of stroke or congestive heart disease confers greater risk than parental history, and the magnitude of association is greater in those with a greater number of affected relatives. ${ }^{14}$

Atherosclerosis is a major cause of carotid artery disease with common risk factors including diabetes mellitus, hypertension, and dyslipidemia. ${ }^{15}$ This was observed in our study in which almost all patients were diabetic, hypertensive, and dyslipidemic. The effectiveness of revascularization and postoperative complications were not related to the history of coronary artery disease of previous myocardial infarction in our cohort. This is different from the results of a study done by Trystuła and Pąchalska ${ }^{16}$ who concluded that comorbidities of atherosclerotic vascular disease, coronary artery disease, and previous myocardial infarction had a significant influence on the effectiveness of revascularization and postoperative health-related quality of life in all patients. In our center, thorough cardiac evaluation including cardiology consultation is mandatory prior to endovascular interventions.

The prevalence of asymptomatic carotid artery stenosis in adults aged 65 years or older is around $0.5-1 \% .^{17}$ The low sample size in our study indicates a low prevalence of carotid artery disease in our community. In a study done by Shaheen et al. ${ }^{2}$ in the central region of Saudi Arabia, the frequency of significant carotid artery disease was only $9 \%$ in their cohort, of which $6.9 \%$ of patients were symptomatic. They concluded that significant carotid artery disease is uncommon occurring in less than $10 \%$ of patients. Our center is a tertiary care referral center in the Western region of Saudi Arabia for the management of complex neurological disorders. A nationwide epidemiological study is warranted to determine the exact prevalence of both symptomatic and asymptomatic carotid artery disease in Saudi Arabia.

Routine screening for carotid artery disease should only be performed for those patients above the age of 65 years with a history of previous stroke and multiple risk factors. Although duplex ultrasonography is a widely available non-in- 
vasive screening tool, the reliability of ultrasonography is questionable. It is an operator-dependent test with variable accuracy rates among healthcare centers. Its use may result in many false-positive tests, especially in a low-prevalence population. For example, in a population of 100,000 adults, screening ultrasonography may result in 940 true-positive and 7,920 false-positive results. This will result in many unnecessary confirmatory tests including conventional cerebral angiography and possible unnecessary interventions. ${ }^{18}$

To perform a successful CAAS, several key steps are considered determinants of the complexity of the procedure and its success. These steps involve gaining access to the common carotid artery, characterizing and crossing the lesion, and deploying the stent. Technical challenges may be encountered during the procedure including high-grade stenosis, complex aortic arch anatomy, difficult femoral artery access, tandem stenotic lesions, circumferential calcification, and internal carotid artery tortuosity. The frequency of these technical challenges was estimated to be $26 \%$. Overcoming these difficulties may be achieved by modifications of the standard technique or conversion to open endarterectomy. ${ }^{19}$ In our study, 2 patients had technical difficulties precluding the success of the CAAS procedure. These difficulties were complete occlusion of the artery (due to delay in performing the procedure with progression of the disease) and aortoiliac occlusion (Leriche syndrome) with no attempt to access the radial artery due to lack of experience from the interventional radiologist.

Postoperative complications for CAAS include stroke, restenosis, and cerebral hyperperfusion syndrome. Cerebral hyperperfusion syndrome may occur in $0.44-11.70 \%$, while intracerebral hemorrhage may occur in $0.36-4.50 \%$. The peak time of cerebral hyperperfusion syndrome is within 12 hours after the procedure (several hours to several days). The periprocedural strokes are usually minor, ipsilateral to the treated artery, and ischemic in nature. A major stroke has a very low overall complication rate (0.6\%). ${ }^{20}$ In our study, 1 patient developed in-stent thrombosis and showering of thrombi with subsequent strokes in the middle cerebral and anterior cerebral arteries likely due to insufficient platelet inhibition despite being on aspirin $325 \mathrm{mg}$ and clopidogrel $75 \mathrm{mg}$ daily 7 days before the procedure. He was known to have a cardiac disease (coronary artery disease with coronary angioplasty). Unfortunately, he died 3 months later from septic shock.

In our study, restenosis was not seen in our patients who were followed up to 5 years. In a study done by Texakalidis et al. ${ }^{21}$, risk factors associated with restenosis were diabetes, dyslipidemia, female gender, renal failure, pretreatment stenosis more than $70 \%$, hypertension, and smoking. Besides the low sample size in our study, our patients undergo aggressive management of all comorbid conditions, which may explain the low rate of restenosis.

The present study had several limitations including a relatively small sample size, being a single-center study, and the retrospective nature of the research. Future multicenter studies with larger sample sizes are warranted to validate our results and determine the frequency of significant carotid artery disease.

\section{CONCLUSION}

Despite advancements in medicine with free healthcare services in Saudi Arabia, the interventional procedures for secondary prevention of strokes are underutilized. Vascular risk factors need to be addressed and managed properly in the Saudi population to reduce the incidence of carotid artery disease and strokes. Protocols similar to other international centers protocols should be adopted including functional outcome scales and multidisciplinary care by different teams should be implemented. Prompt discussion of each case by a board of experts should be activated.

\section{Fund}

None.

\section{Ethics Statement}

This study was approved by the Institutional Review Board (IRB) of King Abdullah International Medical Research Center under IRB number IRBC/0676/18.

\section{Conflicts of Interest}

The authors have no conflicts to disclose.

\section{Author Contributions}

Concept and design: HA and BS. Analysis and interpretation: HA, BS, ASA, AS, and AA. Data collection: BS and ASA. Writing the article: HA, BS, AS, SAA, and AA. Final approval of the article: all authors. Statistical analysis: BS, ASA, and AS. Overall responsibility: HA. 


\section{ORCID}

Hussein Algahtani: https://orcid.org/0000-0001-9484-9838

Bader Shirah: https://orcid.org/0000-0001-6493-2155

Abdullah S. Alghamdi: https://orcid.org/0000-0002-3915-3536

Ahmad Subahi: https://orcid.org/0000-0002-2032-2993

Saeed A. Alqahtani: https://orcid.org/0000-0001-8156-6839

Abdulrahman Alshamy: https://orcid.org/0000-0002-5683-0981

\section{REFERENCES}

1. Katan M, Luft A. Global burden of stroke. Semin Neurol 2018;38:208-211

2. Awada A, al Rajeh S. The Saudi stroke data bank. Analysis of the first 1000 cases. Acta Neurol Scand 1999;100:265-269

3. Lu J, Wang D. Advances in endovascular therapy for ischemic cerebrovascular diseases. Chronic Dis Transl Med 2016:2:135-139

4. Langhoff R. Carotid stenosis - basing treatment on individual patients' needs. Optimal medical therapy alone or accompanied by stenting or endarterectomy. Vasa 2018;47:7-16

5. Moresoli P, Habib B, Reynier P, Secrest MH, Eisenberg MJ, Filion KB. Carotid stenting versus endarterectomy for asymptomatic carotid artery stenosis: a systematic review and meta-analysis. Stroke 2017;48:2150-2157

6. Sardar P, Chatterjee S, Aronow HD, Kundu A, Ramchand P, Mukherjee D, et al. Carotid artery stenting versus endarterectomy for stroke prevention: a meta-analysis of clinical trials. J Am Coll Cardio/ 2017:69:2266-2275

7. Arhuidese IJ, Rizwan M, Nejim B, Malas M. Outcomes of primary and secondary carotid artery stenting. Stroke 2017;48:30863092

8. Safian RD. Asymptomatic carotid artery stenosis: revascularization. Prog Cardiovasc Dis 2017;59:591-600

9. Shaheen MA, Albelali AA, AlKanhal RM, AlSaqabi MK, AlTurki $\mathrm{RM}, \mathrm{AlAskar} \mathrm{RS}$, et al. Frequency, risk factors, and outcomes in patients with significant carotid artery disease admitted to King Abdulaziz Medical City, Riyadh with ischemic stroke. Neurosciences (Riyadh) 2019;24:264-268

10. Elsharawy MA. Carotid endarterectomy in high-risk Arab patients. Interact Cardiovasc Thorac Surg 2009;8:100-103
11. Clinical alert: benefit of carotid endarterectomy for patients with high-grade stenosis of the internal carotid artery. National Institute of Neurological Disorders and Stroke Stroke and Trauma Division. North American Symptomatic Carotid Endarterectomy Trial (NASCET) investigators. Stroke 1991;22:816-817

12. European Carotid Surgery Trialists' Collaborative Group. MRC European carotid surgery trial: interim results for symptomatic patients with severe (70-99\%) or with mild (0-29\%) carotid stenosis. Lancet 1991;337:1235-1243

13. Marulanda-Londoño E, Chaturvedi S. Carotid stenosis in women: time for a reappraisal. Stroke Vasc Neurol 2016;1:192-196

14. Khaleghi M, Isseh IN, Jouni H, Sohn S, Bailey KR, Kullo IJ. Family history as a risk factor for carotid artery stenosis. Stroke 2014:45:2252-2256

15. Rincon F, Sacco RL, Kranwinkel G, Xu Q, Paik MC, Boden-Albala $B$, et al. Incidence and risk factors of intracranial atherosclerotic stroke: the Northern Manhattan stroke study. Cerebrovasc Dis 2009;28:65-71

16. Trystuła M, Pąchalska M. Comorbidities and health-related quality of life following revascularization for asymptomatic critical internal carotid artery stenosis treated with carotid endarterectomy or angioplasty with stenting. Med Sci Monit 2019;25:47344743

17. Jonas DE, Feltner C, Amick HR, Sheridan S, Zheng ZJ, Watford DJ, et al. Screening for asymptomatic carotid artery stenosis: a systematic review and meta-analysis for the U.S. Preventive Services Task Force. Ann Intern Med 2014;161:336-346

18. Mortimer R, Nachiappan S, Howlett DC. Carotid artery stenosis screening: where are we now? Br J Radio/ 2018;91:20170380

19. Choi HM, Hobson RW, Goldstein J, Chakhtoura E, Lal BK, Haser $\mathrm{PB}$, et al. Technical challenges in a program of carotid artery stenting. J Vasc Surg 2004;40:746-751

20. Kim NY, Choi JW, Whang K, Cho SM, Koo YM, Kim JY. Neurologic complications in patients with carotid artery stenting. J Cerebrovasc Endovasc Neurosurg 2019;21:86-93

21. Texakalidis P, Tzoumas A, Giannopoulos S, Jonnalagadda AK, Jabbour P, Rangel-Castilla L, et al. Risk factors for restenosis after carotid revascularization: a meta-analysis of hazard ratios. World Neurosurg 2019;125:414-424 